\title{
Acessibilidade em ambiente virtual de aprendizagem
}

\section{RESUMO}

Albino Szesz Junior albinoir12@gmail.com

Universidade Tecnológica Federal do Paraná, Ponta Grossa, Paraná, Brasil.

Sani de Carvalho Rutz da Silva sani@utfpr.edu.br

Universidade Tecnológica Federal do Paraná, Ponta Grossa, Paraná, Brasil.

Maria Salete Marcon Gomes Vaz salete@uepg.br

0000-0001-9172-1863

Universidade Estadual de Ponta Grossa, Paraná, Brasil.

Dênia Falcão de Bittencourt denia@gmail.com

0000-0002-5883-8602

Universidade Estadual de Ponta Grossa,

Paraná, Brasil.
O objetivo deste trabalho é expor as alterações focadas em acessibilidade virtual realizadas no ambiente virtual de aprendizagem da Universidade Estadual de Ponta Grossa. O trabalho foi realizado em três etapas: na primeira realizou-se estudos de acessibilidades em ambientes virtuais de aprendizagem, na segunda realizou-se alterações no ambiente Moodle versão 2.6 através da instalação de plug-ins e na terceira alterou-se o formato e layout do material disponibilizado. Os resultados apresentados demonstram as mudanças realizadas, tais como, a disponibilização da ferramenta de lupa, alto contraste e leitor de conteúdo, a possibilidade de tradução automática do português para libras, além da padronização de disciplinas. Conclui-se que as ações realizadas satisfazem o objetivo proposto, e destaca-se que encontrar uma interface adequada, com todos os aspectos de usabilidade e acessibilidade satisfeitos é um grande desafio.

PALAVRAS-CHAVE: LMS MOODLE. Tecnologia. Inovação. 


\section{INTRODUÇÃO}

Os avanços das Tecnologias de Informação e Comunicação (TIC) geraram aplicações nas mais diversas áreas do conhecimento, destacando-se a educação como uma das áreas de maior crescimento. Esta evolução permitiu que ocorresse uma revolução na Educação a Distância (EAD).

A partir deste progresso e com o passar do tempo, a presença da internet no cotidiano aumentou e, a EAD se consolidou, tornando-se um cenário para a efetivação da inclusão, pois utiliza as mídias digitais como canal de propagação, minimizando barreiras de acesso, principalmente as geográficas. No entanto, ela ainda tem sido incipiente em promover a inclusão e a integração entre alunos, possivelmente pelas estratégias de ensino ou mesmo pela padronização dos ambientes adotados (PIVETTA, et al.,2014).

Os ambientes que são necessários para realizar o apoio pedagógico a alunos e professores, chamados de Ambientes Virtuais de Aprendizagem (AVAs), são um conjunto de softwares integrados e adequados para oferecer a interface de aprendizado para o aluno e que também proporcionam a multiplicação da informação, além da interação entre alunos e professores, de forma rápida e dinâmica.

Para que esta interação aconteça de forma simples e eficiente, não se pode deixar de lado as discussões quanto à acessibilidade dos Ambientes Virtuais de Aprendizagem (AVA) e as características dos seus usuários, visto que são pontos cruciais para a inclusão de pessoas com deficiências, tema que possui ampla abrangência por estar relacionado aos processos de aprendizagem de significativa cota $(23,9 \%)$ da população nacional segundo o IBGE (2010).

Neste crescente, afim de satisfazer uma das premissas da inclusão social, que é o acesso à educação, a Universidade Estadual de Ponta Grossa (UEPG), através do seu Núcleo de Tecnologia e Educação Aberta à Distância (NUTEAD), começou em 2015, a trabalhar questões de acessibilidade virtual em seu ambiente virtual de aprendizagem.

Diante disso, o objetivo deste trabalho é expor as alterações focadas em acessibilidade virtual realizadas no AVA da Universidade Estadual de Ponta Grossa.

\section{REVISÃO DA LITERATURA}

Segundo Saraiva (2010), a maioria dos cursos realizados na modalidade à distância ou na modalidade semipresencial utiliza a internet, através de sites desenvolvidos para esse fim, os ambientes virtuais de aprendizagem, cuja principal função é a de servir de repositório de conteúdo e meio de interação/comunicação entre os atores envolvidos no processo de ensinoaprendizagem (COLE e FOSTER, 2008), e que possuem diferentes formas de apresentação de suas ferramentas, com funções específicas e maneiras distintas de interação com os usuários facilitando o acesso dos alunos em todas as propostas de conteúdos e atividades (SEIXAS et al.,2012).

Várias ferramentas foram desenvolvidas e comercializadas para esse fim e em razão do custo elevado, variando em função do quantitativo de alunos, grupos de pesquisa desenvolveram plataformas gratuitas. Paiva (2010) destaca alguns 
ambientes, tais como MOODLE, AulaNet, TeleEduc, E-Proinfo, ROODA, Eureka, Virtus, entre outros. Cada plataforma possui características específicas e uma diversidade de ferramentas.

O MOODLE - Modular Objetct-Oriented Dynamic Learning Environment (Módulo Orientado a Objetos de Ambiente Dinâmico de Aprendizagem) é um software livre de administração de conteúdo educacional, para cursos online, utilizados nas categorias de cursos de graduação semipresencial, à distância, extensão, pós-graduação e afins com suporte a diversas plataformas, tais como Unix, Linux, Windows, Mac OS, além de sistemas com suporte à Linguagem PHP, e estando disponível em mais de 40 idiomas.

Com a popularização da internet e a preocupação em aumentar a interação entre homem e computador, foi necessário criar interfaces amigáveis, fazendo com que a usabilidade assumisse um papel relevante, que no entendimento de Varanda (2010) "se refere simplesmente à "facilidade ou dificuldade que um aparato tem em ser operado".

A usabilidade e a acessibilidade são características que agregam qualidade a um produto conteúdo digital, a primeira visa satisfazer um público específico, definido como o consumidor que se quer alcançar quando se define o projeto do produto, o que permite que se trabalhe com as peculiaridades adequadas a esse público-alvo (associadas a fatores tais como a faixa etária, nível socioeconômico, gênero e outros). Já a segunda é que permitirá com que a base de usuários projetada, seja alcançada em sua máxima extensão e que os usuários que se deseja conquistar com o produto tenham êxito em iniciativas de acesso ao conteúdo digital em uso (TORRES; MAZZONI, 2004).

Segundo o World Wide Web Consortium (W3C, 2015), a acessibilidade virtual está relacionada à capacidade de sua utilização por pessoas com deficiência, permitindo que estes usuários sejam capazes de perceber os conteúdos, compreendê-los, realizar atividades de navegação e interação, bem como criar conteúdo na web. Entretanto, os sites de forma geral, apresentam barreiras de acessibilidade, sendo que estas variam conforme o perfil do usuário a acessá-lo.

Afim de evitar essas barreiras, existem recomendações de acessibilidade, tais como a WCAG (WCAG20, 2008). As WCAG (Web Content Accessibility Guidelines) são recomendações de acessibilidade para conteúdo da Web, ou seja, são diretrizes que explicam como tornar o conteúdo Web acessível a todas as pessoas.

Essas recomendações foram desenvolvidas pelo consórcio W3C através do WAI (Iniciativa de Acessibilidade na Web) (WAI, 2015), em colaboração com pessoas e organizações em todo o mundo e hoje encontram-se na versão 2.0.

No Brasil o Governo Federal disponibiliza o eMAG - Modelo de Acessibilidade em Governo Eletrônico (eMAG, 2014), que contém as recomendações de acessibilidade a serem seguidas nos sítios e portais do governo brasileiro. O eMAG está na versão 3.1 e foi desenvolvido tomando como base a WCAG 2.0, documentos internacionais de acessibilidade, pesquisas e o auxílio de pessoas com deficiência.

Esse modelo, eMAG, consiste em um conjunto de recomendações de acessibilidade que são a tradução de boas-práticas que visam tornar o conteúdo Web acessível a todas as pessoas com deficiência, destinando-se aos autores de páginas, projetistas de sítios e aos desenvolvedores de ferramentas para criação 
de conteúdo. A observação destes padrões também facilita o acesso ao conteúdo da Web, independente da ferramenta utilizada (navegadores Web para computadores de mesa, laptops, telefones celulares, ou navegador por voz) e de certas limitações técnicas, como, por exemplo, uma conexão lenta, a falta de recursos de mídia, etc (LOBATO et al., 2012).

Sendo assim, garantir a acessibilidade na Web, é permitir que qualquer indivíduo, utilizando qualquer tecnologia de navegação, visite qualquer sítio e obtenha completo entendimento das informações contidas nele, além de ter total habilidade de interação.

\section{MATERIAIS E MÉTODOS}

O trabalho foi desenvolvido em 2015 no AVA da UEPG, o qual se baseia no learning management system (LMS) Moodle, versão 2.6 e é mantido pelo Núcleo de Tecnologia e Educação Aberta à Distância (NUTEAD). As ações dividem-se em três etapas principais.

A primeira etapa consiste em estudos detalhados de acessibilidade em ambientes virtuais de aprendizagem, além de guias e especificações de acessibilidade recomendados pela W3C e pelo eMAG.

A segunda etapa corresponde as alterações realizadas no LMS Moodle, através da instalações e configuração dos plug-ins e scripts: plug-in Accessibility versão 2.2.2 (AHUJA, 2015), plug-in oohoo - Text to Speech versão 1.0.6 (THIBAUDEAU; BRETIN, 2014), plug-in Onetopic format (BERNAL, 2014) e script Hand Talk (HAND TALK, 2015) para sites.

A terceira etapa corresponde ao formato e layout do material disponibilizado através do AVA, o qual é formatado através do recurso "livro" e do tipo de curso "tópico único", ambos disponibilizado no LMS MOODLE e que possibilitam concentrar as informações através de seções disponibilizadas em um sumário, simulando capítulos de um livro, para então apresenta-las em conjunto com as demais informações da disciplina em um uma interface com layout de abas.

\section{RESULTADOS E DISCUSSÕES}

Através da instalação dos plug-ins Accessibility (AHUJA, 2015) e oohoo - Text to Speech (THIBAUDEAU; BRETIN, 2014) no LMS MOODLE utilizado como AVA na UEPG, disponibilizou-se a ferramenta de lupa e de alto contraste no bloco Acessibilidade (Figura 1), tal bloco permite aos usuários personalizar o AVA às suas necessidades visuais, possibilitando a mudança de tamanho de texto e esquemas de cores, tal como o alto contraste.

Já o bloco Leitor de Conteúdo (Figura 1) possibilita que o usuário ouça todo o conteúdo disponibilizado dentro do AVA ao invés de realizar a leitura, por meio da tecnologia text to speech, o texto é convertido em áudio de forma automática, através de métodos computacionais. 
Figura 1 - Bloco Acessibilidade e Bloco Leitor de Conteúdo
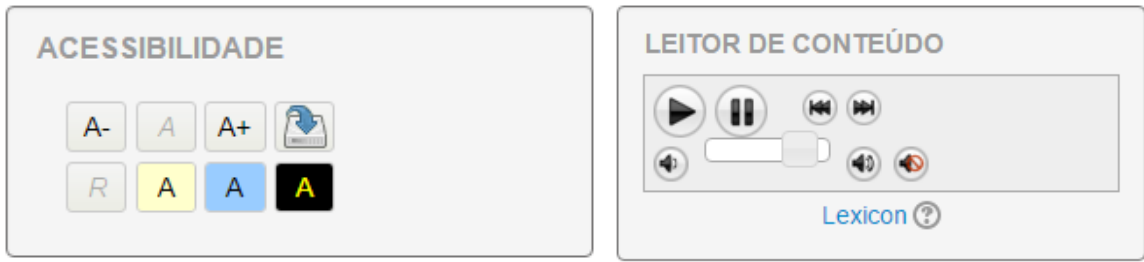

(Fonte: Elaborada pelo Autor)

Outra modificação no AVA foi a inserção do aplicativo Hand Talk para sites (Figura 2), o qual, através da inserção de um script de programação, insere um botão de acessibilidade, possibilitando a tradução de conteúdos textuais em português para Libras, automaticamente.

Figura 2 - Aplicativo Hand Talk em execução no LMS MOODLE da UEPG AVA Graduaçãa sites Académico Bibliotecas Alino Szesz Jun

\section{3 - Teorias da Administração Pública}
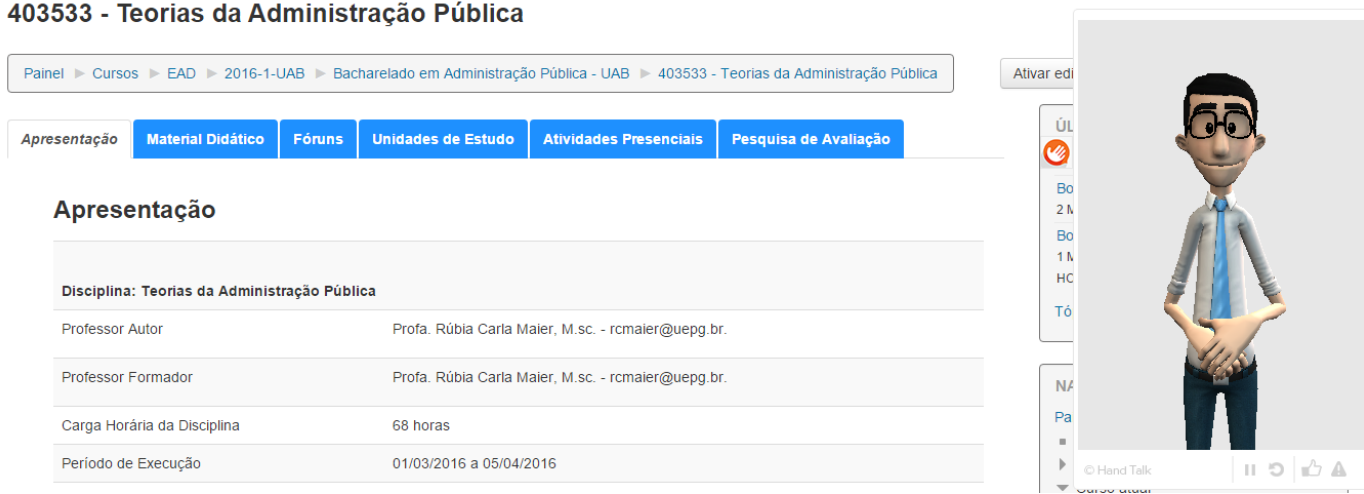

(Fonte: Elaborada pelo Autor)

No formato e layout das disciplinas, destaca-se que até 2013 as disciplinas virtuais da UEPG, baseavam-se em tópicos, onde cada um corresponderia a uma unidade de estudo e a um período de tempo para estudos, neste padrão todas as informações eram expostas na tela de uma só vez, o que sobrecarregava o aluno e o próprio sistema computacional, pois as páginas web demoravam mais tempo para serem carregadas, além de demandarem uma maior banda de internet, uma vez que todo o conteúdo deveria ser carregado e apresentado, isso poderia desmotivar os alunos na sua utilização (Figura 3 (a)).

Na mudança ocorrida no AVA, alterou-se o formato das disciplinas, utilizando rótulos mais atrativos e concentrando as informações dentro da ferramenta "livro" (Figura 3 (b)). Dessa forma, as seções dividem-se em um sumário e em links, otimizando o carregamento e facilitando a navegação dos usuários e a leitura das páginas. 
Figura 3 - Formato de unidade em 2011 (a); Formato de unidade em 2014 (b)

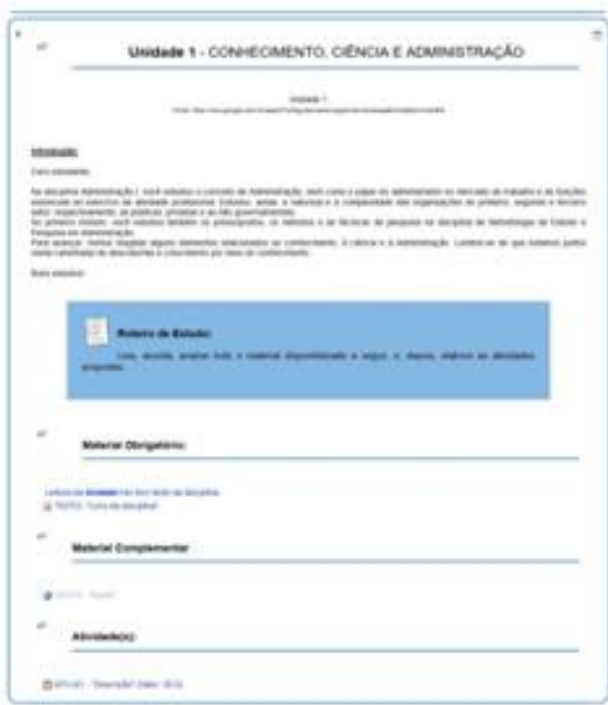

(a)
UNIDADE 1 - Conhecimento, Ciência e Administraçäo

U1A1 - Atividade (Valor: 10,0)

SUMÁRIO

< Sair do livro

1 Introduçäo

2 Roteiro de Estudo

3 Material Obrigatório

4 Material Complementar

5 Atividades Online

6 Acesse as unidades

(Fonte: Elaborada pelo Autor)

Através da instalação do plug-in Onetopic format, o formato de curso alterouse, utilizando-se abas ao invés de tópicos (Figura 4). As unidades de estudo permanecem implementadas através do recurso livro.

Figura 4 - Formato de ABAS com unidades de estudo inseridas via recurso Livro.

\begin{tabular}{|l|l|l|l|l|l|l|l} 
Apresentação & Material Didático & Fóruns & Unidades de Estudo & Atividades Presenciais & Pesquisa de Avaliação
\end{tabular}

UNIDADE 1 - Introdução ao estudo da Administração Pública

Atividade 1 - Questionário (Valor: 5,0)

Atividade 2 - Relato Sobre o Video (Valor: 10,0)

UNIDADE 2 - Administração Pública e suas tipologias

Atividade 3 - Questionário (Valor: 5,0 )

Atividade 4 - Sobre Burocracia (Valor: 10,0)

(Fonte: Elaborada pelo Autor)

A utilização de abas permite uma melhor navegação entre os conteúdos, sua associação com o formato livro, já implementado, possibilita ao usuário a identificação das seções de estudo, além de proporcionar páginas web com carregamento mais rápido.

$\mathrm{Na}$ aba material didático (Figura 5), os materiais da disciplina foram readequados, sendo disponibilizados nos formatos PDF, MP3 e Daisy, além do tradicional livro impresso. 
Figura 5 - ABA Material Didático com a disponibilização de diferentes formatos.

\begin{tabular}{|l|l|l|l|l|l|}
\hline Apresentação & Material Didático & Fóruns & Unidades de Estudo & Atividades Presenciais & Pesquisa de Avaliação
\end{tabular}

\section{Material Didático}

Caro aluno, nesta seção, será disponibilizado o livro didático da disciplina em outros três formatos, PDF, MP3 e Daisy.

Para saber mais sobre esses formatos acesse o link abaixo "Entenda os Formatos".

Esse material possui o mesmo conteúdo do livro impresso

Entenda os Formatos

\section{PDF}

Aqui encontra-se o livro completo da disciplina em formato PDF.

Livro - Teorias da Administração Pública

\section{Áudio Book}

Aqui encontram-se os áudios de cada unidade de seu livro impresso.

Ћ. Áudio Book - Parte 1: PREFÁCIO, APRESENTAÇÃO - Formato MP3 1.1Mb Arquivo de áudio (MP3)

(Fonte: Elaborada pelo Autor)

Importante salientar que os livros em PDF são idênticos aos livros impressos, assim como o MP3 e Daisy, onde os arquivos também são criados com o conteúdo dos livros impressos, tornando-os em "livros falados" pelo computador.

\section{CONSIDERAÇÕES FINAIS}

O objetivo deste trabalho foi expor as alterações focadas em acessibilidade virtual realizadas no AVA da UEPG. Neste contexto, atingiu-se os objetivos propostos surgindo novas possibilidades de pesquisa e desenvolvimento.

As medidas focadas na acessibilidade virtual, ainda são recentes, porém, de acordo com o feedback e pesquisas qualitativas em andamento, já se demonstra como positiva, caracterizando o grau de maturidade do ensino à distância da UEPG.

A principal contribuição deste trabalho é a aplicação dos conceitos de acessibilidade em softwares robustos e de área específica, demonstrando teoria e prática, bem como a produção de conhecimento científico, disseminando práticas já realizadas com sucesso.

Ressalta-se que encontrar uma interface adequada com todos os aspectos de usabilidade e acessibilidade satisfeitos é um grande desafio. Definir as métricas para avaliar e propor interfaces é algo complexo que demanda tempo e um conhecimento aprofundado sobre o assunto.

Como proposta de trabalhos futuros propõem-se a implantação dos conceitos de acessibilidade em todos os cursos virtuais da instituição, bem como uma análise completa das iniciativas já realiza das no âmbito da acessibilidade virtual. Pretendese também a publicação de novos trabalhos demonstrando outras ações de acessibilização da instituição. 


\title{
ACCESSIBILITY IN VIRTUAL LEARNING ENVIRONMENT
}

\begin{abstract}
The purpose of this work is to describe the changes focused on virtual accessibility performed on the virtual learning environment of Ponta Grossa State University. The work was accomplished in three stages: the first focused on studying accessibility in virtual learning environments; in the second one, the Moodle version 2.6 environment was modified through plug-ins installation; finally, in the third stage the format and layout of the available material were updated. The results show the performed modifications, such as the availability of a loupe tool, high contrast and content player, and the possibility of automatic translation from Portuguese into Libras, besides the standardization of disciplines. It is concluded that the actions taken meet the proposed objective, and highlight the major challenge of finding an appropriate interface with all aspects of usability and content accessibility.
\end{abstract}

KEYWORDS: Distance Education. LMS MOODLE. Technology. Innovation. 


\section{REFERÊNCIAS}

AHUJA, H. Accessibility. Versão 2.2.2. Software. Moodle, 2015.

BERNAL, D. H. Onetopic Format. Software. Moodle, 2015.

COLE J., FOSTER H. Using MOODLE: Teaching with the Popu-lar Open Source Course Management System. 2. ed. Estados Unidos: O’ReiLLY; 2008.

eMAG - Modelo de Acessibilidade em Governo Eletrônico, 2014. Disponível em:< http://emag.governoeletronico.gov.br/>. Acesso em: 16 de fev 2016.

HAND TALK. Hand Talk. Disponível em: < https://www.handtalk.me/sites/>. Acesso em: 12 junho 2015.

IBGE. Censo 2010. Disponível em: < http://censo2010.ibge.gov.br/>. Acesso em: 23 set 2015.

LOBATO, F. H., et al. Modelo de Acessibilidade em Governo Eletrônico.

Disponível em: <http://www.gestaoti.org/SISP/clad/lobatofe.pdf>. Acesso em: 10 fev 2016.

PAIVA, V.M.O; Ambientes virtuais de aprendizagem: implicações epistemológicas. Revista Educação em Revista, Belo Horizonte, v. 26, n. 3, dez. 2010.

PIVETTA, E. et al. Surdos e acessibilidade: análise de um ambiente virtual de ensino e aprendizagem. Rev. bras. educ. espec., Marília , v. 20, n. 1, p. 147-162, 2014.

SARAIVA, Karla; Educação a distância: outros tempos, outros espaços. Ponta Grossa: UEPG, 2010. 246p.

SEIXAS, C. A. et al.. Ambiente virtual de aprendizagem: estruturação de roteiro para curso online. Revista Brasileira de Enfermagem - REBEn. Brasília. V.65. p.660-666. jul-ago. 2012.

THIBAUDEAU, P., BRETIN, N. oohoo - Text to Speech. Versão 1.0.6. Moodle. 2014. v. 33, n. 2, dez. 2004. ISSN 1518-8353. 
VARANDA, R. C.; et al. Construção e Validação da Escala de Reações à Interface Gráfica para Cursos de Educação a Distância. Revista Psicologia: Teoria e

Pesquisa V. 26, p. 371-380. 2010.

W3C, World Wide Web Consortium. Disponível em: < http://www.w3.org>. Acesso em: 25 abril 2015.

WAl, Web Accessibility Initiative. Disponível em: < http://www.w3.org/WAl/>. Acesso em: 25 abril 2015.

WCAG20, Web Content Accessibility Guidelines 2.0 - W3C Recommendation 11 December 2008. Disponível em: <http://www.w3.org/TR/WCAG/>. Acesso em: 25 abril 2015.

DOI: 10.3895/rbect.v10n1.5692

Como citar: JUNIOR, A. S.; SILVA, S. C. R.; VAZ, M. S. M. G.; BITTENCOURT, D. F. Acessibilidade em ambiente virtual de aprendizagem. Revista Brasileira de Ensino de Ciência e Tecnologia, v. 10, n. 1, 2017. Disponível em: <https://revistas.utfpr.edu.br/rbect/article/view/5692>. Acesso em: xxx.

Direito autoral: Este artigo está licenciado sob os termos da Licença Creative Commons-Atribuição 4.0 Internacional.

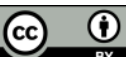

